\title{
Debates sobre Tecnología y Educación: Caminos contemporáneos y conversaciones pendientes
}

\section{(Debates regarding Technology and Education: contemporary pathways and pending conversations)}

\author{
Linda Castañeda \\ Universidad de Murcia, UM (España) \\ Coordinadora del Monográfico
}

DOI: http://dx.doi.org/10.5944/ried.22.1.23020

\section{Como referenciar este artículo:}

Castañeda, L. (2019). Debates regarding Technology and Education: contemporary pathways and pending conversations. RIED. Revista Iberoamericana de Educación a Distancia, 22(1), pp. 29-39. doi: http://dx.doi.org/10.5944/ $\underline{\text { ried.22.1.23020 }}$

\section{Resumen}

El objetivo de este número especial de RIED es contribuir a promover procesos críticos de transformación en el ámbito de la Tecnología Educativa, a través de una colección de nueve artículos que consiguen hacer, a la vez, un estado de la cuestión y un análisis de algunos de los debates que toman fuerza en el campo en los últimos tiempos.

Esta introducción, por su parte, no pretende resumir dichos artículos, sino ofrecer una visión de conjunto y, al mismo tiempo, proponer un diálogo que incluya los puntos de vista expresados en esos artículos, otras perspectivas que seguramente nos han sido insinuadas en investigaciones e, incluso, otras que parecen latentes en nuestra praxis. En concreto, enunciamos sucintamente, pero con ánimo propositivo, siete conversaciones pendientes que se refieren a: 1) Entender los procesos educativos con tecnología y las tecnologías para entender procesos educativos; 2) Tomar decisiones educativas con tecnología; 3) Agencia digital; 4) La naturaleza de las instituciones educativas en tiempos de tecnología; 5) Enriquecer el papel de los participantes en las instituciones con tecnologías; 6) Definir la tecnología educativa y, por último, 7) Investigar en tecnología educativa.

Palabras clave: tecnología educativa; investigación educativa; agencia digital; alfabetización en datos. 


\begin{abstract}
The main goal of this special issue of RIED is trying to contribute to promoting critical transformation processes in the field of Educational Technology through a collection of nine articles that manage to make, at the same time, a state of the art and an analysis of some of the debates that have gained strength in the field in recent times.

This introduction is not intended to summarize these articles, but to offer an overview and, at the same time, to propose a dialogue that includes the points of view expressed in these articles, other perspectives that have surely been insinuated to us in research and even others that seem latent in our praxis. Specifically, with an intention of proposition, seven pending conversations are succinctly considered, that refer to: 1 ) Understanding educational processes with technology and technologies to understand educational processes; 2) Making educational decisions with technology; 3) Digital agency; 4) The nature of educational institutions in times of technology; 5) Enriching the role of participants in institutions with technologies; 6) Defining educational technology; and, finally, 7) Investigating educational technology.
\end{abstract}

Keywords: educational technology; educational research; digital agency; data literacy.

Situar, aunque sea parcialmente, algunos de los temas que marcan la actualidad de la investigación sobre el impacto de la tecnología en educación es una tarea importante para emprender procesos críticos de transformación. Intentar compilar en una publicación con la trayectoria de la Revista Iberoamericana de Educación a Distancia un número especial que sirva a ese cometido es una tarea difícil y una inmensa oportunidad de reflexión educativa que ha dado como fruto el volumen que tienen ante sus ojos.

En este número de la revista se han conseguido juntar en un mismo espacio de lectura, reflexióny debate; nueveartículos deprimer nivel, firmados por investigadores de muy diversos contextos (geográficos, epistemológicos y profesionales), en los que se abordan de forma diversa algunas de las preocupaciones que la investigación en el campo de la tecnología educativa vive en nuestros días.

Si bien se trata de textos que abordan cuestiones que podrían considerarse "asentadas" en el abanico temático de la Tecnología Educativa, lo cierto es que muchos de ellos se aproximan a tales temas de forma innovadora, especialmente afirmativa o directamente problematizadora. Algunos, incluso, lo hacen de todas esas formas a la vez.

Sin embargo, no introduciremos, a continuación, en detalle esos artículos, les recomendamos vivamente la lectura de sus resúmenes si quieren tal sinopsis, y el contenido entero de todos ellos si lo que quieren es unirse a la discusión. Por el contrario, hemos considerado que lo más pertinente es aprovechar la oportunidad para intentar ofrecer una visión de conjunto y, al tiempo, abrir un diálogo con esos puntos de vista que se han expresado en los documentos que hemos reunido, con 
otros que seguramente nos han sido insinuados en investigaciones e incluso con otros que parecen latentes en nuestra praxis.

\section{ALGUNOS CAMINOS Y DEBATES}

Hacer un alto para enunciar las conversaciones pendientes y los debates contemporáneos en tecnología educativa (en adelante TE), no se trata tanto de delimitar de qué deberíamos hablar hoy cuando abordamos, en profundidad, la relación entre la tecnología y la educación, sino más bien se trata de remarcar y apuntar cuáles son aquellos puntos calientes del debate que, bien por nuestro afán de avance, bien por lo difícil de la delimitación del objeto (tanto lo que respecta a la tecnología como a la educación) bien por la intensidad y rapidez con la que se desarrollan las mismas tecnologías e impactan y reconfiguran la realidad social, parecen habernos pasado más desapercibidos de lo deseable en los últimos tiempos.

Si bien está claro que no son los únicos, a continuación, se proponen algunos enunciados que creemos podrían resultar de interés cuando apagamos los destellos de colores de lo fulgurante y actual, y afinamos la vista en lo importante; aquellos que sobresalen a la luz de la investigación y subyacen como constante preocupación en nuestros debates formales e informales.

Si nos empeñáramos en una pregunta que guiara el análisis y la enunciación de esos temas, la pregunta sería algo como ¿de qué hablamos en tecnología educativa? Creemos que, para empezar, podríamos hablar de:

\section{Entender procesos educativos con tecnología y tecnologías para entender procesos educativos}

Uno de los asuntos que ha irrumpido con mayor fuerza -y polémica- en la relación entre educación y tecnología, en nuestros días, es el tema de los datos y su utilización en el mundo educativo. La capacidad extraordinaria que tienen las tecnologías desde hace relativamente pocos años de registrar, obtener, almacenar y operar con datos discretos sobre la actividad de las personas ha resultado un campo de indiscutible interés en los últimos años y que conocemos, a nivel general, como analíticas del aprendizaje (Learning Analytics, en adelante LA).

Seguramente, y muy en la línea de las preocupaciones iniciales de los modelos explicativos de las LA (al respecto recomendamos ciertamente revisar el modelo de Clow, 2012), uno de los debates que deberían abordarse con algo más de rigor debería ser el relacionado con ¿Hasta qué punto los datos que nos proporcionan las tecnologías son relevantes para la educación?, o mejor aún, ¿Hasta qué punto el operar con esos datos nos da información realmente valiosa para el desarrollo de los procesos educativos? (Buckingham Shum et al., 2016).

Algunos de los desarrollos que se están haciendo en la actualidad y que, afortunadamente, han encontrado su hueco en este número especial, superan la ya 
clásica promesa inicial de la automatización de los procesos educativos (Bartolomé, Castañeda, y Adell, 2018) y tienen que ver más con la capacidad de las personas para utilizar esos datos en su beneficio, como vía para entender mejor esos procesos (de lo que tenemos un buen ejemplo en el artículo presentado en este trabajo por parte de Ullman, De-Liddo, y Bachler).

Como hemos dicho, se trata de abordar cómo las tecnologías -en este caso las LA- pueden contribuir, por un lado, al desarrollo cognitivo y por otro, al aprovechamiento de las cada vez más complejas dinámicas de relación que, de forma inédita, podemos establecer en estos entornos. Pero no se trata de investigar cómo hacer que la tecnología desarrolle la cognición o aproveche las dinámicas (cosa que ya nos ha ocupado bastante), sino de cómo proponer desde la tecnología herramientas que apuesten por explorar esas nuevas formas de trabajo y ofrezcan a las personas visiones nuevas o alternativas de la realidad que construyen en sus entornos de discusión (a través, por ejemplo, de visualizaciones de esos procesos), pero huyendo de simplificaciones reduccionistas.

Tenemos nuevos procesos, formas inéditas de vivir asuntos como la participación en red, la co-creación de contenido y de debates, pero seguimos prestando poca atención a la estructura organizativa que soporta tales procesos. Dedicamos poco esfuerzo a reconstruir cualitativamente estrategias didácticas comprometidas con ellos y, menos aún, a la formación de aprendices y organizaciones en las habilidades y actitudes para trabajar en profundidad con estos flujos de información y sacarles provecho.

\section{Tomar decisiones educativas con tecnología}

De la misma manera, y también mediante las LA, otros desarrollos que marcan esos caminos por transitar en TE se basan en la idea de que un sujeto más informado de sus posibilidades para aprender y de su realidad (al menos de lo que los datos dicen de esa realidad), podría gestionar y desarrollar mejor su Entorno Personal de Aprendizaje (PLE) a lo largo de su vida y de acuerdo con sus prioridades, en la línea de lo que nos proponen Attwell y Hughes en su artículo.

Esos desarrollos se refieren, por un lado, a la necesidad de transparencia en los algoritmos para los usuarios de las aplicaciones, algo que empieza a distar mucho de lo posible teniendo en cuenta que dicha transparencia parece no ser siquiera patrimonio de los mismos desarrolladores, quienes - por la complejidad de esas cadenas de toma de decisiones que programan- resultan no ser del todo dueños conscientes del camino de sus ingenios.

Pero, por otro lado, esos "avances" y sus posibilidades exigen que se forme a las personas en la lectura y aprovechamiento de esos datos, en el desarrollo de una competencia complementaria a lo "digital" que tenga que ver con su alfabetización en datos (data literacy). Esta formación sería casi ineludible en nuestros tiempos no solo para profesionales (Carlson y Johnston, 2015), sino para todos los ciudadanos y 
debería permitirles, no solo entender las estadísticas (Gummer y Mandinach, 2015), sino también hacerles críticos en su utilización (Pangrazio y Selwyn, 2018).

Una competencia basada no solo en el acceso y la utilización optimizada de los datos (en la línea del quantified self del que viene hablándose casi desde el principio de los tiempos de las LA (Swan, 2013 y Whitson, 2013), sino también de una aproximación personal y socialmente sostenible (no en vano empezamos a poner cada vez más énfasis en los enfoques de slow media y tech (Patrignani y Whitehouse, 2018; Rauch, 2018). Una competencia que despeje esa sensación de oscuridad y turbidez que rodea a la utilización de herramientas automáticas que traten los datos que producimos en las herramientas telemáticas y nos ayuden -sí, a las personas que aprendemos- a potenciar nuestras posibilidades de toma de decisiones autónoma y enriquecida.

\section{Agencia digital}

Todo esto pone de relieve uno de los conceptos sobre los que hemos sobrevolado en los últimos tiempos y que, tras ser más visible por el espacio que deja en los análisis realizados que por su consideración explícita, resulta probablemente una de las claves del empoderamiento posible de las personas en los nuevos tiempos digitales: el concepto de agencia digital.

Definida recientemente de forma sencilla como "la capacidad de controlar y adaptarse al mundo digital" (Passey et al., 2018, p. 426), la agencia digital, como la agencia misma, resulta complementaria a cualquier propuesta de competencia que hagamos, no en vano la agencia define la capacidad de toma de decisiones consciente y comprometida que hacen las personas (Emirbayer y Mische, 1998).

Eso, en tiempos donde preguntas como: ¿Quién -o qué- toma las decisiones sobre lo que queremos y empezamos a aprender?, ¿son nuestros profesores, nosotros mismos, las empresas para las que trabajamos o que nos ofrecen servicios, el algoritmo con el que nuestras redes sociales nos ofrecen contenido, etc.?, resultan difíciles de responder, debería interpelarnos directamente en términos educativos. De igual manera: ¿Hasta qué punto estamos comprometidos con que una mayor capacidad de acceder y manejar los datos de las tecnologías móviles está aumentando la agencia de las personas en este mundo digital? ¿En qué medida asumimos la responsabilidad en el ámbito educativo de que nuestros intereses de investigación y discurso deberían apostar por hacer conscientes y responsables de su proceso de toma de decisiones a las personas, en tanto que dueños del proceso de enseñanza y aprendizaje? ¿Hasta qué punto la agencia -digital y analógica - tienen una concreción curricular? Incluso más, ¿̇La sociedad y la escuela promueven este empoderamiento y no solo el desarrollo de habilidades instrumentales en el manejo de la tecnología? Resulta un camino cuya definición y complejidad no deberíamos eludir. 


\section{La naturaleza de las instituciones educativas en tiempos de tecnología}

Estas preocupaciones no son exclusivamente individualistas, ni tampoco aluden solo al campo mismo de la TE como ente más o menos abstracto. Las instituciones educativas, y su redefinición actual, resultan cruciales en tanto que organismos sujeto y objeto de cambios promovidos por la tecnología.

Vivimos en permanentes crisis identitarias de nuestras instituciones (Williamson, 2013; Masschelein y Simons, 2015), en la que algunos de los debates sobre quién gobierna, qué y para qué, no terminan de estar bien definidos y, por lo tanto, tampoco suficientemente desarrollados.

Como bien nos recuerdan en su artículo de esta colección los profesores Moreira Teixeira, Bates, y Mota, la gobernanza de nuestras instituciones, su papel situado y cada vez menos equiparable o generalizable a otras (en tanto que organizaciones que van más allá de la provisión de "servicios" educativos) y su cada vez más necesario perfil de desarrollo social-comunitario nos interpela sobre cómo podemos conseguir que la tecnología obre en favor de esas instituciones y que no les redefina acríticamente.

\section{Enriquecer el papel de los participantes en las instituciones con tecnologías}

Nos encontramos en tiempos en que las grandes esperanzas venidas de desarrollos en aspectos como la utilización por parte de los estudiantes de herramientas para apoyar su trabajo habitual en las instituciones educativas (como en la experiencia que nos presentan Dabbagh, Fake, y Zhang) o de posibilidades pedagógicas para la implementación de didácticas concretas (bien perfiladas en el documento de Trujillo, Salvadores, y Gabarrón), se ven siempre matizadas por la crítica y casi bajo sospecha.

No en vano, otros desarrollos supuestamente educativos, consecuencia de los cambios aparentemente tecnológicos, resultan de políticas rápidas deimplementación de un tipo de asunto, que, como en el caso del pensamiento computacional (tratado en profundidad en el trabajo de Adell, Llopis, Esteve, y Valdeolivas de este número), tiene una carga ideológica no explicitada pero pesada y evidente, que repercute de forma obvia en el curriculum y no siempre de la manera en que nos gustaría.

Nos movemos en la dualidad de intentar dar pasos en la mejora delas competencias de los participantes de esas instituciones para el aprovechamiento del momento tecnológico que les rodea (como revela el trabajo que se incluye en la colección sobre competencia digital del profesorado que presentan Durán, Prendes, y Gutiérrez), pero, al mismo tiempo, hablamos de cómo conseguir que esos mismos participantes no vean más troceada aún su aproximación al conocimiento y que no sean meros 
operarios avanzados de la maquinaria tecnológica que les rodea. Buscamos sujetos que tengan -o mejor, asuman- un papel socialmente comprometido.

El inmenso papel político que ejercen las tecnologías también en educación (Winner, 1980), la forma en que condicionan nuevas y complejas formas de alfabetización de las personas y configuran modelos culturales y de pensamiento que no pueden ser obviadas (Martínez Bonafé, 2001; Hull y Stornaiuolo, 2014), los modelos de interacción entre tecnología y sociedad (Bromley, 1997) y entre cienciatecnología y sociedad, nos exigen una mirada mucho más comprometida sobre la relación entre educación y tecnología, que la que busca, a semejanza de la ingeniería, la mejora de la educación con el uso de uno u otro conocimiento.

\section{Definir la tecnología educativa}

Y todo lo anterior, todos los debates propuestos interrogan, como reflexionará Khün en su artículo, forzosamente a la investigación en educación y a la tecnología educativa de forma directa.

En los últimos tiempos, algunos autores han llamado la atención sobre cómo, a pesar de haber sido conscientes de la evolución en el concepto de tecnología, los planteamientos que desarrollan la tecnología educativa (tanto como campo de estudio, como práctica o como ámbito de investigación), siguen entendiéndola desde un punto de vista primordialmente instrumental, siempre desde perspectivas tremendamente deterministas (Webster, 2017; Adell, 2018) y cada vez más empobrecidas (Oliver, 2016).

La TE de la que hablamos ha superado con mucho a la que se ha descrito en los estudios evolutivos clásicos (Area, 1991; Cabero, 2007; de Pablos, 2009). Ya no son los recursos audiovisuales de los años 40 del siglo pasado, desarrollados por los militares para la formación en masa de sus tropas, ni son los MOOC actuales (Liyanagunawardena, Adams, y Williams, 2013; Vázquez-Cano y Meneses, 2015), etc., bueno, no sólo.

Tampoco se reduce a las máquinas de enseñanza programada como las de Skinner en los años 50, ni con la enseñanza asistida por ordenador (EAO) de los 70, ni siquiera con cosas como Knewton y otras modernidades de aprendizaje adaptativo, etc. ni siquiera eso es suficiente para abarcarlo (Bartolomé et al., 2018). No son tampoco los medios de comunicación de masas y sus revoluciones en colaboración con la televisión educativa o los satélites en los años 80 y 90 (Cabero y Martínez Sánchez, 1995), ni tampoco son solamente los medios de comunicación uno a uno, soportados en redes telemáticas, ni los medios 2.0, personalizados -al menos en teoría- hasta el extremo, omnipresentes en dispositivos que pueblan los bolsillos de la inmensa mayoría de la población, ni los contenidos transmedia distribuidos en diversas plataformas y promoviendo la participación del espectador (Scolari, 2018).

Hablamos de un ente más amplio que todas esas tecnologías juntas y más específico que todas esas tecnologías por separado (Cabero, Barroso, y Llorente, 
2015). Y hablamos de entenderlas desde sus complejidades, contradicciones y retos. Y todo eso, en educación, teniendo en cuenta que la relación entre los dos conceptos que sustentan la tecnología educativa (tecnología y educación) se ha revelado como sistémica y compleja.

Las dicotomías entre tecnodeterminismo y sociodeterminismo, incluso de aquellas discusiones sobre si una u otra tecnología funciona o no (Cuban, 1993; Clark, 1994), aunque siguen ocupando parte de nuestra energía, se han revelado como insuficientes a la hora de comprender el efecto de la tecnología en la educación.

La cuestión es que la tecnología, al menos entendida de forma amplia, ni incide en la educación solo cuando es "nueva", ni se "aplica" a la educación, ni se "implementa" o "integra" en ella, ni siquiera se enriquece con ella, eso es solo una parte de lo que la tecnología hace en educación.

Estos tiempos, el movimiento que los caracteriza, la naturaleza de sus realidades y las fuerzas que los condicionan, nos reclaman una visión más clara de nuestra relación con la realidad educativa y con la realidad social (e indefectiblemente tecnológica). Una visión que ponga en valor nuestra relación con lo verdaderamente importante, lo que nos da sustento epistemológico y social, una relación que deje de pensar puntualmente en contextos o en aparatos y se comprometa globalmente con personas y sociedad. Una visión que seguramente pase por redefinir claramente los límites de qué es la tecnología educativa hoy, y cómo son sus relaciones con el resto de ciencias de la educación y con otras disciplinas afines.

\section{Investigar en tecnología educativa}

Esa realidad, a la vez, reclama de nosotros apuestas decididas referidas a la investigación.

Los caminos que transitamos y los retos que nos plantean las situaciones actuales exigen de la TE una investigación más interdisciplinar, con verdaderos diálogos y controversias multi-área resueltas en conjunto, no solo consensos interesados y superficiales. Reclaman una investigación más ambiciosa, que se atreva con diseños más interesantes, en los que se otorgue más protagonismo a los investigados y se saque verdadero provecho de los nuevos códigos (no siempre para convertirlos en textos y números). Se nos demanda una investigación mejor contada y de mayor recorrido, que recupere otros debates y temas educativos de primer nivel y aporte valor en ellos (en el sentido del análisis propuesto por Castañeda, Tur, y TorresKompen en su artículo sobre PLE incluido en este número) y, por lo mismo, que tenga una clara vocación de impacto en la realidad educativa.

En último término, una investigación -y una reflexión- que vaya más allá del optimismo posibilista, la ingenuidad solucionista o el pesimismo ilustrado de los últimos años (Morozov, 2013; Castañeda, 2016; Selwyn, 2016; Castañeda y Selwyn, 2018) y apueste por un realismo comprometido con algo que sobrepase la propia investigación como actividad. 


\section{CONCLUSIONES}

Como decíamos más arriba, no son todos los caminos ni enunciamos todos los debates. Es más, sabemos que muchos se han tratado antes. Estamos ante una conversación larga y preferiblemente pausada en la que esperamos que el lector participe... mientras lo lee, en un debate cara a cara, en publicaciones y que, en el futuro, entre todos podamos seguir profundizando.

Este documento solo pretende ser una provocación, fruto de las sugerentes aportaciones que se pueden encontrar en el número especial que presentamos y, paralelamente, fruto de la motivación misma de esta compilación.

Nuestra intención no es solo hacer un ejercicio de mera crítica (que ya hemos venido realizando algunos en los últimos tiempos) y que resulta de todo punto necesario, pero insuficiente, sino también resultar propositivos e intentar hacer apuestas decididas por definir dónde queremos ir y por qué caminos queremos transitar, independientes - que no ajenos- a la vorágine propia de los tiempos que corren y que hace esos caminos y esos horizontes, cada vez, más difusos.

\section{REFERENCIAS}

Adell, J. (2018). Más allá del instrumentalismo en tecnología educativa. En J. Gimeno (Ed.), Cambiar los contenidos, cambiar la educación. Madrid: Morata.

Area, M. (1991). Los medios, los profesores y el currículo. Barcelona: Sendai.

Bartolomé, A., Castañeda, L., y Adell, J. (2018). Personalisation in educational technology: the absence of underlying pedagogies. International Journal of Educational Technology in Higher Education, 15(1). doi: https://doi. org/10.1186/s41239-018-0095-0

Bartolomé, A., y Moral, J. M. (Eds.) (2018). Blockchain en Educación. Cadenas rompiendo moldes. Barcelona: LMI (Colección Transmedia XXI). Recuperado de http://www.lmi.ub.es/transmedia21/

Bromley, H. (1997). The Social Chicken and The Technological Egg: Educational Computing and the Technology/Society Divide. Educational Theory, 47(1), 5165. doi: https://doi.org/10.1111/j.17415446.1997.00051.x
Buckingham Shum, S., Sándor, Á., Goldsmith, R., Wang, X., Bass, R., y McWilliams, M. (2016). Reflecting on Reflective Writing Analytics: Assessment Challenges and Iterative Evaluation of a Prototype Tool. En Proceedings of the Sixth International Conference on Learning Analytics y Knowledge (pp. 213-222). New York, NY, USA: ACM. doi: https://doi. org/10.1145/2883851.2883955

Cabero, J. (Ed.). (2007). Tecnología Educativa. Madrid: McGraw-Hill Interamericana de España S.L.

Cabero, J., Barroso, J., y Llorente, M. del C. (2015). Tecnología educativa: historia, concepto y bases conceptuales. En J. Cabero y J. Barroso (Eds.), Nuevos retos en tecnología educativa (19-40). Madrid: Síntesis.

Cabero, J., y Martínez Sánchez, F. (Eds.) (1995). Nuevos canales de comunicación en la enseñanza. Madrid: Editorial Universitaria Ramón Areces.

Carlson, J., y Johnston, L. (Eds.) (2015). Data Information Literacy: Librarians, Data, 
and the Education of a New Generation of Researchers. West Lafayette, Indiana: Purdue University Press. Recuperado de http://www.jstor.org/stable/j.ctt6wq2vh

Castañeda, L. (2016). Suelo y sol: más pensamiento y contexto para la innovación educativa. Boletín de la Institución Libre de Enseñanza, (104), 37-50.

Castañeda, L., y Selwyn, N. (2018). More than tools? Making sense of the ongoing digitizations of higher education. International Journal of Educational Technology in Higher Education, 15(22). doi: https://doi.org/10.1186/s41239-0180109-y

Clark, R. E. (1994). Media will never influence learning. Educational Technology Research and Development, 42(2), 21-29. doi: https://doi.org/10.1007/ BF02299088

Clow, D. (2012). The Learning Analytics Cycle: Closing the Loop Effectively. En Proceedings of the 2nd International Conference on Learning Analytics and Knowledge (pp. 134-138). New York, NY, USA: ACM. doi: https://doi. org/10.1145/2330601.2330636

Cuban, L. (1993). Computers meet classroom: Classroom wins. Teachers College Record, 95(2), 185-210.

de Pablos, J. (2009). Tecnología educativa: La formación del profesorado en la era de Internet. Málaga: Ediciones Aljibe, S.L.

Emirbayer, M., y Mische, A. (1998). What is agency? American Journal of Sociology, 103(4), 962-1023. doi: https://doi. org/10.1086/231294

Gummer, E., y Mandinach, E. (2015). Building a Conceptual Framework for Data Literacy. Teachers College Record, 117(4), 1-22. Recuperado de http:// www.tcrecord.org/library/abstract. asp? contentid $=17856$

Hull G. A., y Stornaiuolo A. (2014). Cosmopolitan Literacies, Social Networks, and "Proper Distance": Striving to Understand in a Global World.
Curriculum Inquiry, 44(1), 15-44. doi: https://doi.org/10.1111/curi.12035

Liyanagunawardena, T. R., Adams, A. A., y Williams, S. A. (2013). MOOCs: A Systematic Study of the Published Literature 2008-2012. International Review of Research in Open and Distance Learning, 14(3), 202-227. doi: https:// doi.org/10.19173/irrodl.v14i3.1455

Martínez Bonafé, J. (2001). Arqueología del concepto «compromiso social» en el discurso pedagógico y de formación docente. Revista Electrónica de Investigación Educativa (REDIE), 3(1). Recuperado de http://www.redalyc.org/ articulo.oa?id=15503106

Masschelein, J., y Simons, M. (2015). Education in times of fast learning: the future of the school. Ethics and Education, 1O(1), 84-95. doi: https://doi.org/10.1080 /17449642.2014.998027

Morozov, E. (2013). To Save Everything, Click Here: The Folly of Technological Solutionism. New York: PublicAffairs.

Oliver, M. (2016). What is Technology. En N. Rushby y D. Surry (Eds.), Wiley Handbook of Learning Technology. (3557). Chichester, UK; Malden, MA: Wiley Handbooks in Education.

Passey, D., Shonfeld, M., Appleby, L., Judge, M., Saito, T., y Smits, A. (2018). Digital Agency: Empowering Equity in and through Education. Technology, Knowledge and Learning, 23(3), 425439. doi: https://doi.org/10.1007/ s10758-018-9384-X

Patrignani, N., y Whitehouse, D. (2018). Slow tech and ICT: a responsible, sustainable and ethical approach. Cham: Palgrave Macmillan.

Rauch, J. (2018). Slow media: why «slow» is satisfying, sustainable, and smart. New York: Oxford University Press.

Selwyn, N. (2016). Is technology good for education? Cambridge, UK: Polity Press.

Swan, M. (2013). The Quantified Self: Fundamental Disruption in Big Data 
Science and Biological Discovery. Big Data, 1(2), 85-99. doi: https://doi. org/10.1089/big.2012.0002

Vázquez-Cano, E., y Meneses, E. L. (2015). La filosofía educativa de los MOOC y la educación universitaria. RIED. Revista Iberoamericana de Educación a Distancia, 18(2), 25-37. doi: https://doi. org/10.5944/ried.18.2.14261

Webster, M. D. (2017). Philosophy of Technology Assumptions in Educational Technology Leadership. Journal of
Educational Technology y Society, 2o(1), 25-36. Recuperado de https://www.jstor. org/stable/jeductechsoci.20.1.25

Whitson, J. R. (2013). Gaming the quantified self. Surveillance and Society, 11(1-2), 163-176.

Williamson, B. (2013). The future of the curriculum: school knowledge in the digital age. London: The MIT Press.

Winner, L. (1980). Do Artifacts Have Politics? Daedalus, 109(1), 121-136.

\section{PERFIL ACADÉMICO Y PROFESIONAL DE LA AUTORA}

Linda Castañeda. Doctora en Tecnología Educativa por la Universitat de les Illes Balears. Profesora del Departamento de Didáctica y Organización Escolar de la Universidad de Murcia y miembro del Grupo de Investigación de Tecnología Educativa (GITE) de la misma universidad. Su investigación se interesa especialmente por el impacto de la tecnología en la educación, la implementación de pedagogías emergentes en contextos formales y no formales, Entornos Personales de Aprendizaje (PLE) y factores clave de desarrollo curricular en el mundo digital. E-mail: lindacq@um.es

Dirección:

Facultad de Educación

Universidad de Murcia

30100 Murcia (España)

Fecha de recepción del artículo: 05/11/2018

Fecha de aceptación del artículo: 05/11/2018

Fecha de aprobación para maquetación: 18/11/2018 